\section{Recent Advances in High-Speed Orientation Mapping}

Matthew M. Nowell ${ }^{1}$, Martina Chui-Sabourin ${ }^{2}$, and John O. Carpenter ${ }^{1}$

${ }^{1}$ EDAX-TSL, Draper, UT

${ }^{2}$ EDAX-TSL, Mahwah, NJ matt.nowell@ametek.com

Orientation mapping via automated analysis of Electron Backscatter Diffraction (EBSD) patterns has developed into an established microstructural analysis tool in the electron microscopy community. From the early 1990s, when this technique became commercially available, there has been a steady increase in the data acquisition rates as shown in Figure 1. Currently, orientation mapping speeds of over 200 analyzed patterns per second have been achieved. With these types of acquisition rates now available, the strategy on how to best use EBSD and orientation mapping has also shifted. Early adopters of this technique had to allocate hours of Scanning Electron Microscope (SEM) beam time in order to collect statistically significant data. With current technology, what was collected in hours can now be obtained in minutes. The goal of this article is to introduce this high-speed orientation mapping and present results illustrating the benefits of this capability.

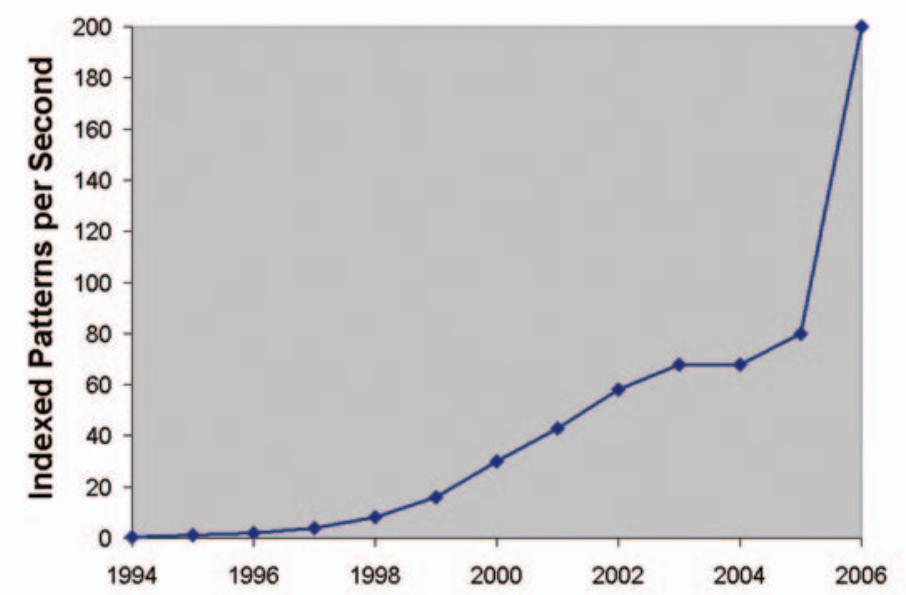
TSL).

Figure 1 - Orientation mapping speed by year (using OIM by EDAX-

To demonstrate high-speed orientation mapping, a sample of 316L stainless steel was selected and carefully prepared for EBSD analysis [1]. With SEM conditions of $20 \mathrm{kV}$ acceleration voltage and an incident beam current of approximately $3 \mathrm{nA}$, EBSD patterns were collected and analyzed at a rate of 200 points per second (pps). Data was collected using a hexagonal sampling grid from a $1850 \mu \mathrm{m} \times 1750 \mu \mathrm{m}$ area with $2 \mu \mathrm{m}$ spacing between measurements, corresponding to approximately 952,000 measurements. The total data collection time was slightly under 80 minutes. The resulting orientation image (EBSD image quality combined with surface normal orientation) is shown in figure 2 (See Figure 5 for the colored stereographic triangle key). The indexing success rate was $99.5 \%$.

In order to obtain these high-speed acquisition rates in combination with this type of indexing success rate, the steps necessary to collect and analyze EBSD patterns must be optimized for speed and accuracy. Take for example the patterns collected from 316L stainless steel shown in Figure 3a-b. Figure 3a shows the EBSD pattern collected at full resolution while Figure $3 \mathrm{~b}$ shows a pattern collected from the same location at a lower resolution. This reduction of resolution is termed binning, and this process allows the CCD within the EBSD detector to generate sufficient frame rates

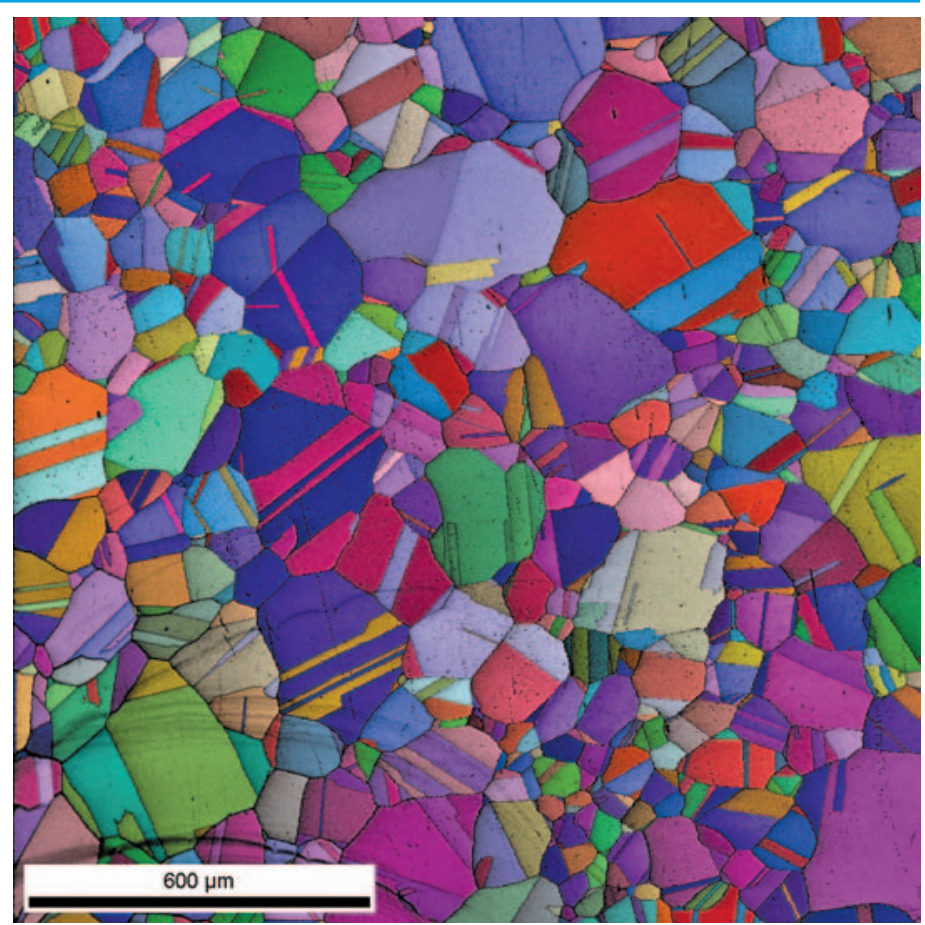

Figure 2 - Combined EBSD image quality map and orientation map (surface normal direction) for $316 \mathrm{~L}$ stainless steel.

to enable high-speed data collection. Of course the patterns must be of sufficient resolution and quality to facilitate accurate and consistent band detection for reliable pattern analysis. The resolution required is often a function of the crystallographic symmetry of the material of interest, as well as sample preparation quality and SEM operating conditions. In addition to camera resolution and frame rates requirements, the EBSD pattern analysis software must be able to detect the diffraction bands and determine the corresponding crystallographic orientation. To obtain high-speed collection, these routines must also perform at speeds of $200 \mathrm{pps}$ or faster. This can require optimization of analysis routines as well as increased computing capability. Ideally a system will match the pattern analysis speed with the frame rate produced by the CCD detector. The closer the match between these two rates, the better the overall efficiency of the system. However, one must be careful not to sacrifice pattern analysis performance in the quest for speed alone.

To investigate the real-world effects of high-speed data sampling strategies, an aluminum metallization film on a silicon substrate was selected. This type of film can be fabricated into interconnect lines for microelectronic devices. An equation to correlate the mean time to failure (MTF) performance of the film under current load as a function of microstructural parameters was given by Vaidya and Sinha as:

$$
M T F=K\left(\frac{S}{\sigma^{2}}\right) \cdot \log \left[I_{(111)} / I_{(200)}\right]^{3}
$$

where $S$ is the average grain size, $\sigma$ is the standard deviation of the grain size distribution, $I_{(111)}$ and $I_{(200)}$ are the orientation densities of the (111) and (200) texture components, and $K$ is a material constant [2]. Orientation mapping is well suited for simultaneously measuring both texture and grain size. Combining this analytical capability with the high-speeds now available for data collection makes orientation mapping more suitable as a process and quality control tool.

Figure 4 shows a Secondary Electron (SE) detector image of the aluminum film at 2,000 $\times$. The film was examined in the asreceived condition. A moderate amount of surface topography can 


\section{Multiphase Materials Characterization \\ Fully Integrated...Totally Seamless}

Powerful Analytical Tools Optimized for Your SEM to Provide Results with Confidence

- Pegasus...Simultaneous data acquisitions for EDS (chemistry) and EBSD (crystallography)Includes the patented Chl-Scan software to improve multiphase analysis capability

- Neptune...Complimentary union of EDS and WDS for superior X-ray microanalysis-Improves quantification especially for low level concentrations

- Trident...Ultimate materials characterization system integrates EDS, EBSD, and WDS with no analytical performance compromise-Provides complete solutions for all your materials characterization requirements

\section{Results with Confidence}

As the world's leader in Electron Beam Microanalysis, EDAX continues to raise the performance standard with innovative features that are easy to adapt and provides results that you can trust to be complete and accurate.

For more information on our Seamless Integrated Systems for EDS, EBSD, and WDS visit our web site at www.EDAX.com/integration or call 1.201.529.4880 Visit us at M \& M Booth 416

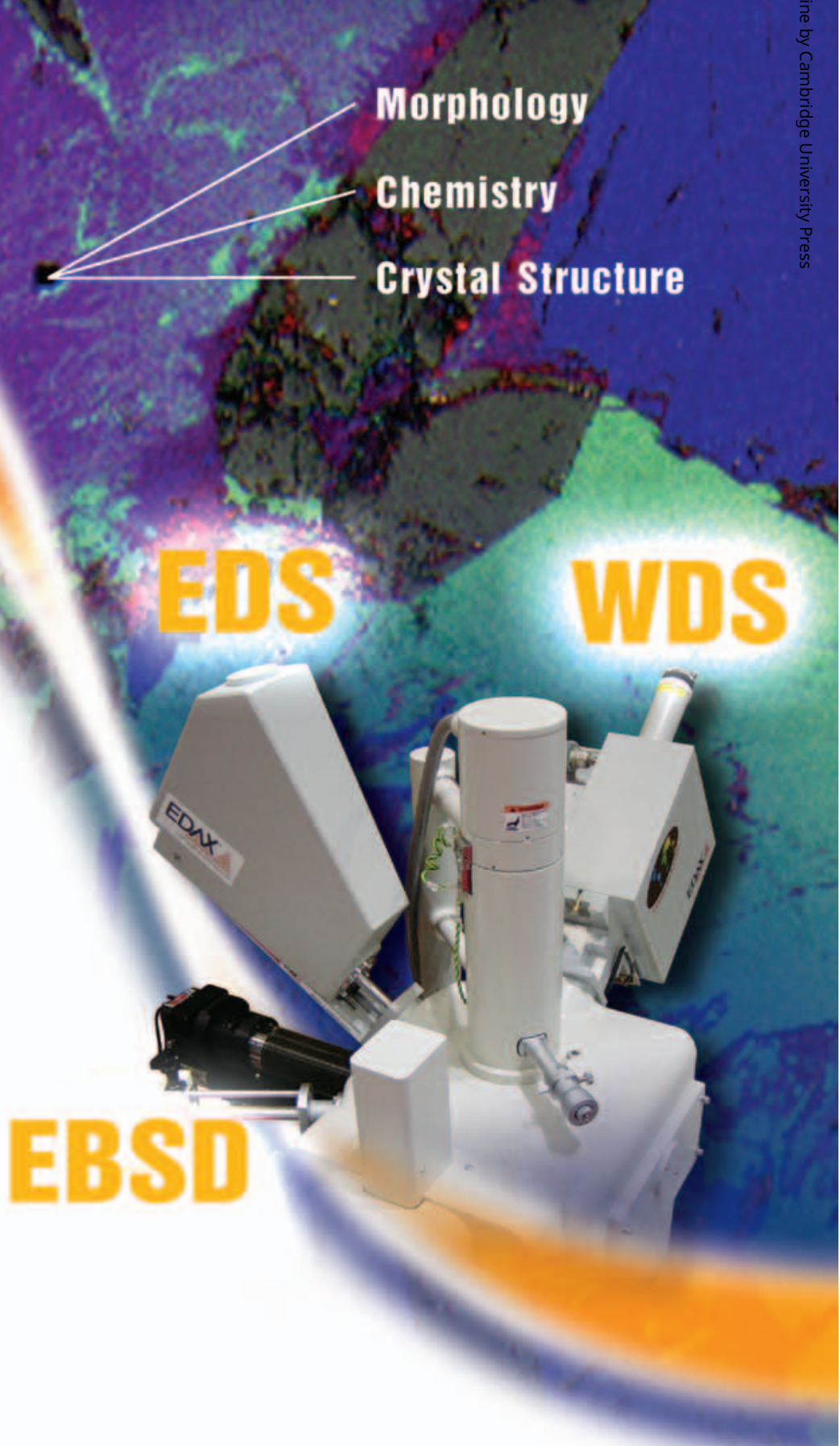

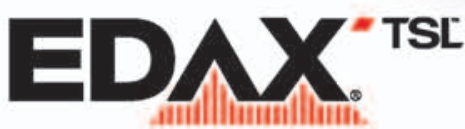

advanced microanalysis solutions 


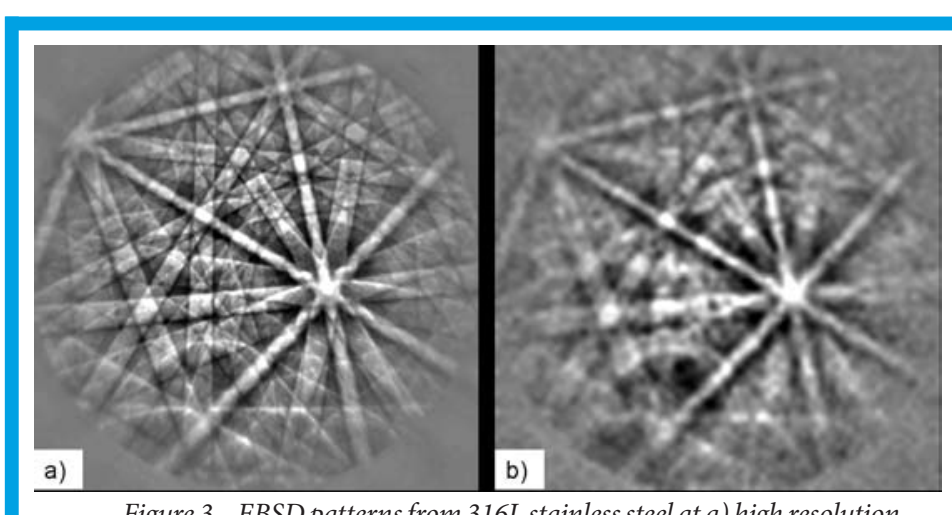

Figure 3 - EBSD patterns from 316L stainless steel at a) high resolution and b) low resolution.

be observed. When applying a standard background correction to enhance EBSD pattern contrast, this topography will introduce slight variations in pattern intensity that can make accurate band detection

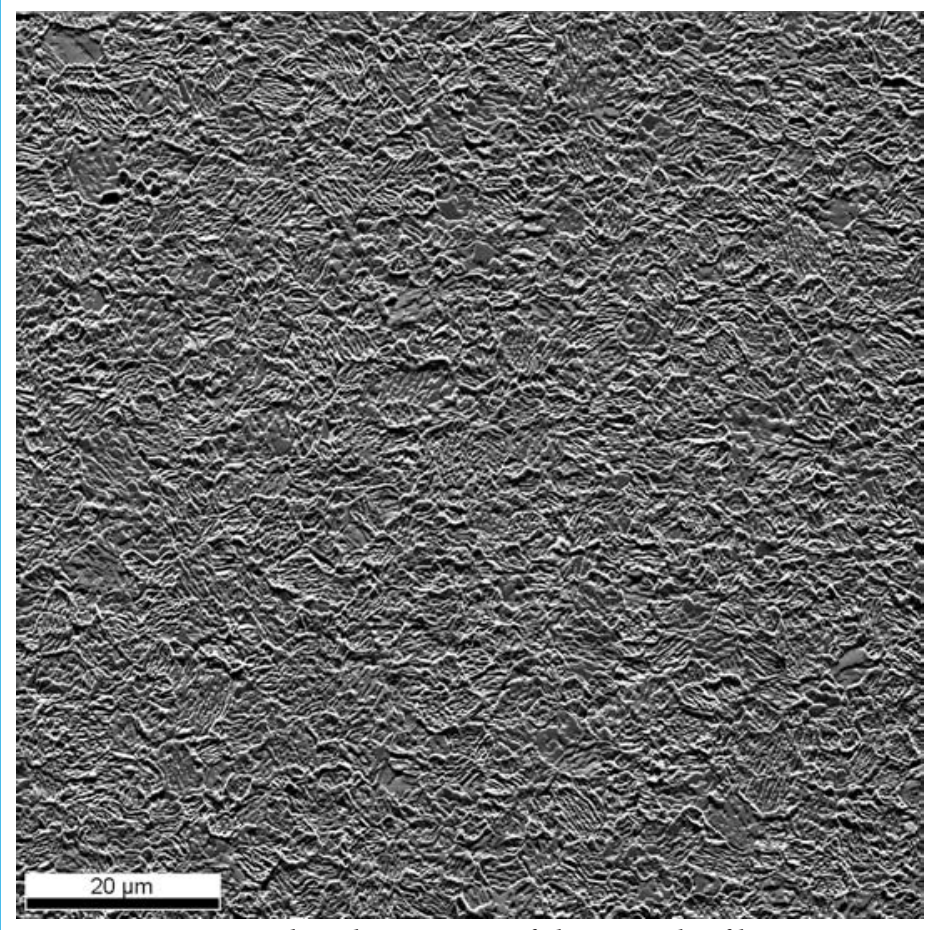

Figure 4-Secondary electron image of aluminum thin film at 2,000X magnification.

more difficult. Under the same SEM analytical conditions as the $316 \mathrm{~L}$ sample, the resulting indexing success rate was $92 \%$. This rate can partially be contributed to the surface topography and partially to the fact that aluminum has a lower atomic number ( $\mathrm{Z}$ number) and therefore produces fewer backscattered electrons. This results in a lower signal level on the EBSD detector, which is compensated

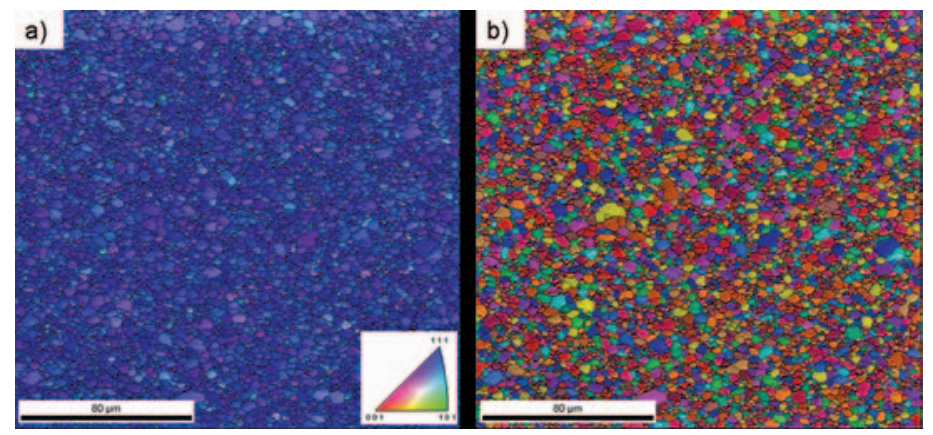

Figure 5 - EBSD image quality maps combined with a) orientation map (surface normal direction) and b) grain map for aluminum thin film area 1 . for with electronic gain within the CCD with a corresponding increase in noise. By increasing the incident beam current from approximately $3 \mathrm{nA}$ to $5.5 \mathrm{nA}$, the same gain level can be used with a corresponding indexing rate of greater than $96 \%$. An informal survey of SEM vendors indicates that general analytical conditions range from 2 to $20 \mathrm{nA}$ of beam current. High-speed orientation mapping is obtainable within this range.

Orientation maps were collected from 5 randomly selected regions at 200pps. Different regions were selected to avoid any contamination effects from a prior map. The scan dimensions, step size between measurements, and total collection time for each area is listed in Table 1. The longest collection time was selected to be approximately 1 hour. This time period was selected because in many facilities SEM time is scheduled by the hour, and this should give an idea of the amount of information that can be collected in that time.

\begin{tabular}{|c|c|c|c|}
\hline \multicolumn{4}{|c|}{ Table 1 - Data collection parameters for aluminum thin film maps } \\
\hline Al Film Maps & $\begin{array}{c}\text { Scan Dimensions } \\
(\mu \mathrm{m})\end{array}$ & Step Size $(\mu \mathrm{m})$ & $\begin{array}{c}\text { Collection } \\
\text { Time }(\mathrm{min})\end{array}$ \\
\hline Area 1 & $208 \times 195$ & 0.25 & 64 \\
\hline Area 2 & $208 \times 195$ & 0.5 & 16 \\
\hline Area 3 & $104 \times 97$ & 0.5 & 4 \\
\hline Area 4 & $75 \times 70$ & 0.5 & 2 \\
\hline Area 5 & $55 \times 52$ & 0.5 & 1 \\
\hline
\end{tabular}

Figure 5 shows an orientation map (image quality combined with surface normal orientation) and a grain map (image quality combined with randomly colored grains) collected from Area 1. Note that the orientation map shows primarily a (111) orientation in the surface normal direction. The grain map is derived by grouping together similarly oriented measurements (within $5^{\circ}$ ) and then randomly coloring the grains to show size and morphology. From the collected data, the average grain size (number average), the total number of grains sampled (excluding edge grains), and the (111) orientation density were calculated. These results are shown in Table 2.

\begin{tabular}{|c|c|c|c|}
\hline \multicolumn{4}{|c|}{ Table 2 - Analytical results from aluminum thin film maps } \\
\hline $\begin{array}{c}\text { Al Film } \\
\text { Maps }\end{array}$ & $\begin{array}{c}\text { Avg. Grain Size } \\
(\mu \mathrm{m})\end{array}$ & $\begin{array}{c}\text { Total Number of } \\
\text { Grains Sampled }\end{array}$ & $\begin{array}{c}\text { (11) Orientation } \\
\text { Density }\end{array}$ \\
\hline Area 1 & 2.34 & 6365 & 15.101 \\
\hline Area 2 & 2.47 & 5949 & 15.305 \\
\hline Area 3 & 2.46 & 1445 & 15.833 \\
\hline Area 4 & 2.38 & 777 & 15.844 \\
\hline Area 5 & 2.41 & 404 & 15.733 \\
\hline
\end{tabular}

The effects of sampling step size relative to grain size can be explored by comparing map areas 1 and 2, where the scan areas are constant, but the step size is varied from $0.25 \mu \mathrm{m}$ to $0.5 \mu \mathrm{m}$. Grain maps from both areas are shown in Figure 6. With an average grain size of approximately $2.4 \mu \mathrm{m}$, a $0.25 \mu \mathrm{m}$ step size will result in about 100 points measured within an average-sized grain while a $0.5 \mu \mathrm{m}$ step will product about 25 points within a grain. This expectation is consistent with experimental observations. The measured grain sizes for each area are reasonably comparable, with the difference between the two less than the smallest step size used. Considering this, a $0.5 \mu \mathrm{m}$ step size was considered adequate for characterization considering the $4 \times$ decrease in acquisition time.

Areas 2-5 were selected to compare the effects of sampling time. While keeping constant an acquisition rate of 200 indexed points per second and a $0.5 \mu \mathrm{m}$ step size, scan areas were selected to produce collection times of $16,4,2$, and 1 minutes. The resulting grain maps are shown in Figure 7 . While the number of grains sampled 


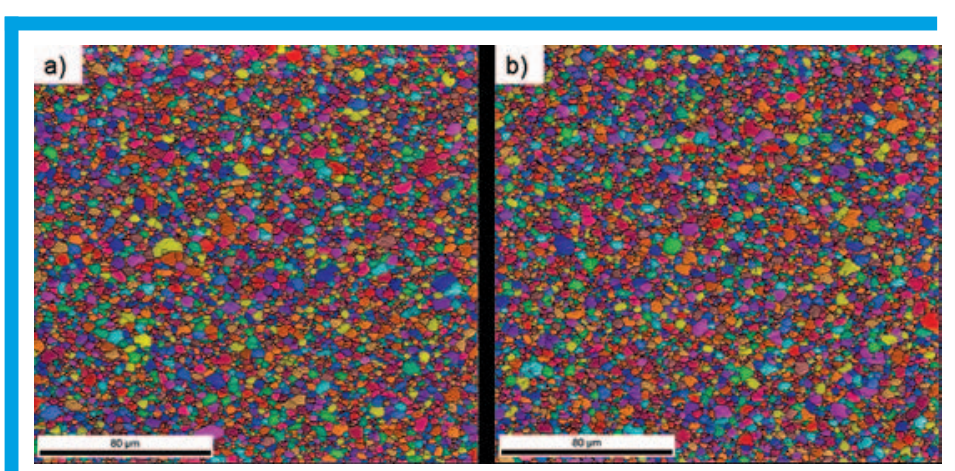

Figure 6- EBSD image quality maps combined with grain maps for a) area 1 with $0.25 \mu \mathrm{m}$ steps and $b$ ) area 2 with $0.5 \mu \mathrm{m}$ steps.

decreases with decreasing mapping time, even in a 1 minute scan over 400 grains are sampled. For a strongly textured material like this aluminum film, this is a statistically significant sampling. For more moderately textured materials (a rolled material for example), 1,000 grains sampled would be adequate for characterization [3]. As a good rule of thumb, as the preferred orientation strength decreases, the number of grains required to characterize that texture increases. However these results show that in 16 minutes, almost 6,000 grains were sampled. The measured grain size and (111) texture strength results are all in good agreement and any could be used as input into the MTF equation described above. One can now imagine applying EBSD-based orientation mapping as a process and quality control tool to monitor grain size and preferred orientation as shown here, as well as other microstructural parameters such as grain shape, grain boundary character distribution, and percent recrystallization.

In summary, the availability of high-speed orientation mapping can produce statistically relevant data in times of 1 hour or less. This could help minimize time pressure on SEMs, allow for rapid data collection in a manufacturing environment, or just enable larger datasets to be collected resulting in better statistics.

\section{References:}

[1] M.M. Nowell, R.A. Witt, and B.W. True, Microscopy Today, Vol 13 (2005) Number $4 \mathrm{p} 44$.

[2] S. Vaidya and A.K. Sinha, Thin Solid Films, 75 (1981) p253.

[3] S.I. Wright and U.F. Kocks, Proceeding of the Eleventh International Conference on Textures of Materials, (1996) p.53.
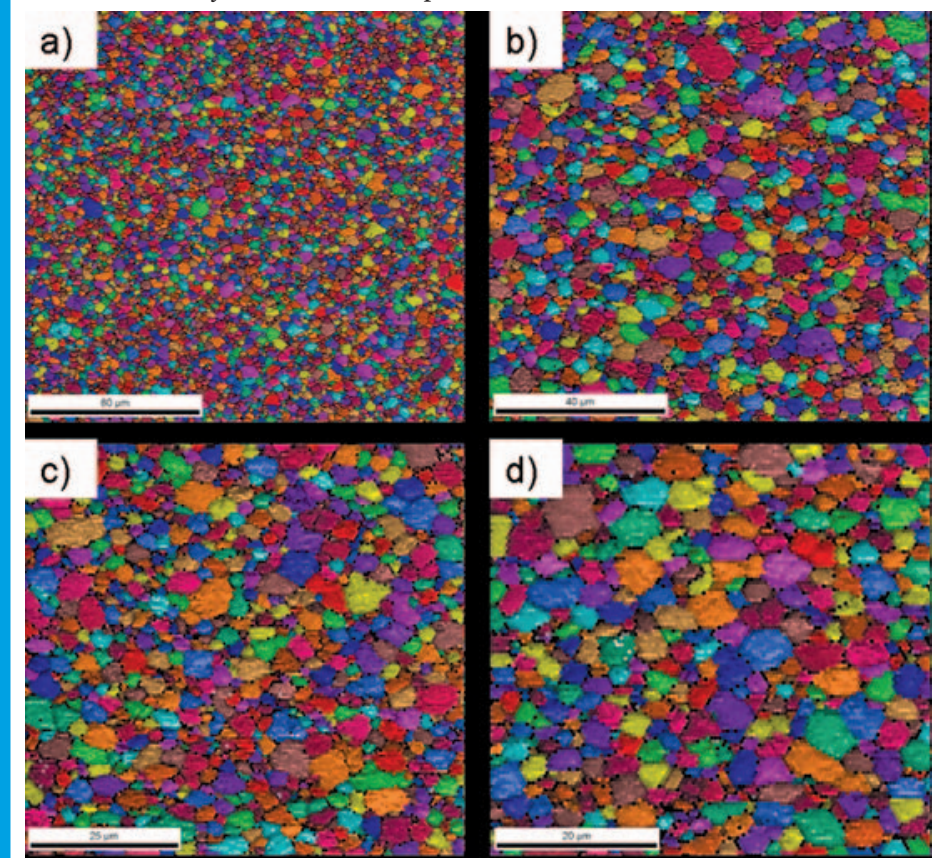

Figure 7-EBSD image quality maps combined with grain maps for a) 16 minute, b) 4 minute, c) 2 minute, and d) 1 minute acquisition times.

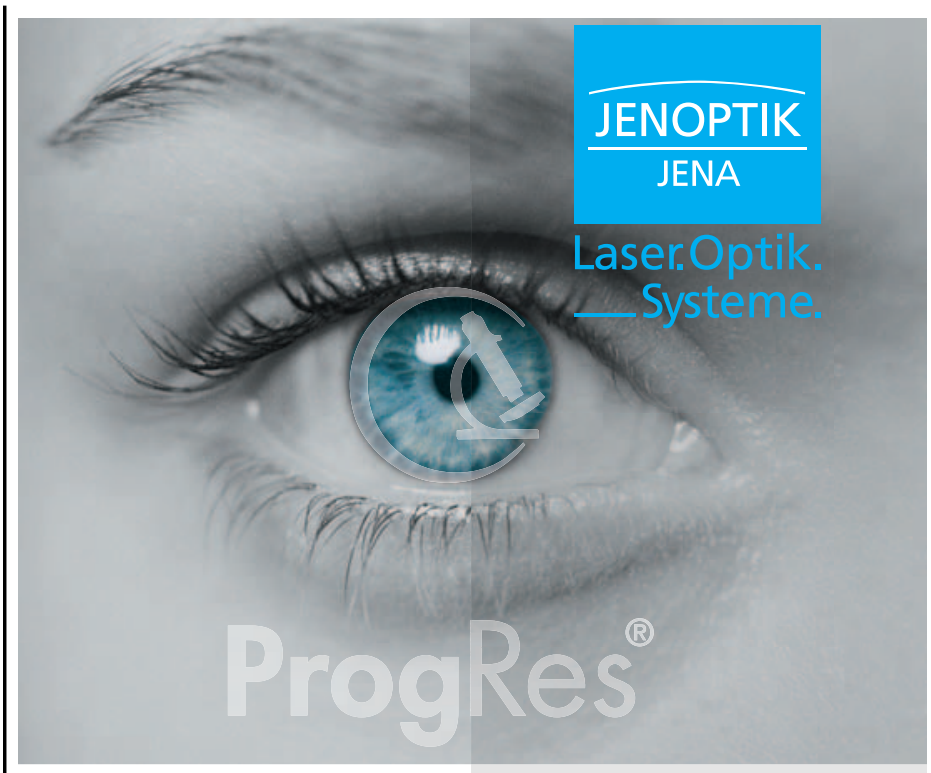

ProgRes ${ }^{\circledR}$ cameras

as digital turnkey-solution

Routine cameras for analysis

and documentation.

- Fast \& high-resolution

cameras for scientific

research.

- Cooled monochrome

cameras for fluorescence.

The ProgRes ${ }^{\circledR}$ CapturePro software 2.1 now offers even more measurement tools and a higher level of userfriendliness.

In combination with easyLab image management system from IMAGIC, ProgRes ${ }^{\circledR}$ cameras are the basis for a turnkey-solution in processing, documentation and presentation of high-quality digital images increasing the efficiency of your lab.

Further information: www.progres-camera.com

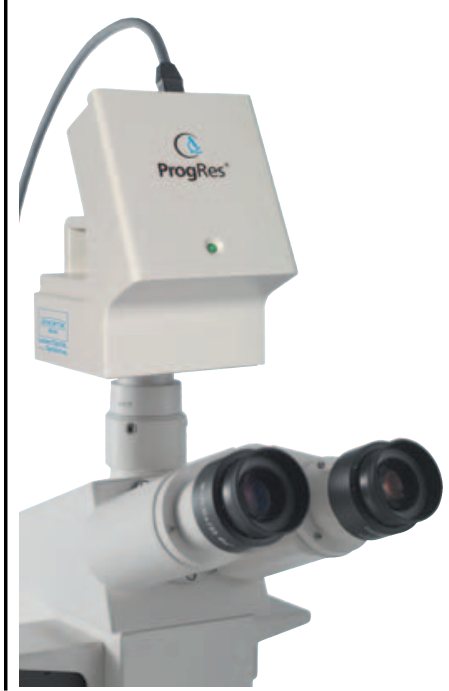

